\title{
ELODIE: A spectrograph for accurate radial velocity measurements
}

\author{
A. Baranne ${ }^{1}$, D. Queloz 2 , M. Mayor ${ }^{2}$, G. Adrianzyk ${ }^{3}$, G. Knispel ${ }^{3}$, D. Kohler ${ }^{3}$, D. Lacroix ${ }^{3}$, J.-P. Meunier ${ }^{3}$, \\ G. Rimbaud ${ }^{3}$ and A. Vin ${ }^{3}$ \\ 1 Observatoire de Marseille, 2 Place Le Verrier, F-13248 Marseille, France \\ 2 Observatoire de Genève, CH-1290 Sauverny, Switzerland \\ 3 Observatoire de Haute-Provence, F-04870 Saint Michel l'Observatoire, France
}

Received September 28, 1995; accepted February 15, 1996

\begin{abstract}
The fibre-fed echelle spectrograph of Observatoire de Haute-Provence, ELODIE, is presented. This instrument has been in operation since the end of 1993 on the $1.93 \mathrm{~m}$ telescope. ELODIE is designed as an updated version of the cross-correlation spectrometer CORAVEL, to perform very accurate radial velocity measurements such as needed in the search, by Doppler shift, for brown-dwarfs or giant planets orbiting around nearby stars. In one single exposure a spectrum at a resolution of $42000(\lambda / \Delta \lambda)$ ranging from $3906 \AA$ to $6811 \AA$ is recorded on a $1024 \times 1024$ CCD. This performance is achieved by using a $\tan \theta=4$ echelle grating and a combination of a prism and a grism as cross-disperser. An automatic on-line data treatment reduces all the ELODIE echelle spectra and computes cross-correlation functions. The instrument design and the data reduction algorithms are described in this paper. The efficiency and accuracy of the instrument and its long term instrumental stability allow us to measure radial velocities with an accuracy better than $15 \mathrm{~m} \mathrm{~s}^{-1}$ for stars up to 9th magnitude in less than 30 minutes exposure time. Observations of 16th magnitude stars are also possible to measure velocities at about $1 \mathrm{~km} \mathrm{~s}^{-1}$ accuracy. For classic spectroscopic studies $(S / N>100) 9$ th magnitude stars can be observed in one hour exposure time.
\end{abstract}

Key words: instrumentation: spectrographs — methods: data analysis — techniques: spectroscopic — radial velocities

\section{Introduction}

ELODIE is the new fibre-fed echelle spectrograph of the $1.93 \mathrm{~m}$-telescope of Observatoire de Haute-Provence (OHP), France. It was put into operation at the end of 1993. This instrument has been designed for three different observing objectives:

- high resolution spectroscopy of relatively bright stars.

- cross-correlation spectroscopy to provide accurate radial velocity measurements.

- accelerometry, this spectrograph being part of the accelerometer proposed by Connes (1985).

These targets have imposed different constraints on the definition of the spectrograph, but constraints coming from accurate Doppler measurements have been the strongest ones. ELODIE has been designed from the beginning as an updated version of the cross-correlation spectrometer CORAVEL (Baranne et al. 1977, 1979). New CCDs of large dimensions and low read-out-noise opened the possibility of replacing the optical correlation between the spectrum and a physical template by a numerical correlation between a CCD spectrum and a "software template". Numerical correlation of high resolution spectra

Send offprint requests to: D. Queloz with CORAVEL-like templates has proven its efficiency, particulary for low signal-to-noise spectra (Dubath et al. 1990; Queloz et al. 1995a; Pont et al. 1995a). The use of such "binary" templates is not optimal in terms of signal analysis. However, the extreme ease of designing templates, selecting only some spectral lines for a specific problem and the extremely smooth (quasi-perfect Gaussian shape) cross-correlation functions computed is so important an advantage that we have maintained this approach for ELODIE.

The efficiency of cross-correlation spectroscopy for radial velocity measurements of late-spectral-type stars (Griffin 1967; Baranne et al. 1979) is well established, but the experience gained with the two CORAVEL since 1977, at the OHP and La Silla Observatories, has shown the limitations of this technique when using a slit spectrograph and a physical template. In particular, the instrumental accuracy is limited to some $200 \mathrm{~m} \mathrm{~s}^{-1}$ (Duquennoy \& Mayor 1991a). This considerably limits the possibility of searching for giant planets or brown dwarfs orbiting around stars. This important limitation is mainly due to errors introduced by the variable illumination of the entrance slit (guiding, focus, variable optical axis of the telescope and calibration lamp, etc.). See Griffin \& 
Griffin (1973) for a discussion of classical difficulties affecting radial velocity measurements. The fixed geometry of the CORAVEL template, being calculated for a well defined air-refractive index, also produces wavelength shifts which are not fully compensated by the zero-point shift determined by the calibration lamp.

The difficulties related to the fixed geometry of the mask used in the optical cross-correlation technique are fully removed with the numerical correlation. Another advantage of the numerical cross-correlation is the possibility of building different templates adapted to different spectral types or measurement goals (see below). An important gain is also expected in the limiting magnitude due to the much better quantum efficiency of the CCD compared to a photomultiplier, and also by avoiding the sequential scanning needed to build the cross-correlation function in CORAVEL-type instruments. In fact, the loss of efficiency due to the scanning is about a factor ten (the ratio between the scanning span and the width of the entrance slit). However, some loss of efficiency is expected from the much larger noise per pixel of the CCD compared to the noise of a single photo-electric detector.

Instruments specialized in precise Doppler shift measurements, in operation for more than a decade, reach accuracies of about $20 \mathrm{~m} \mathrm{~s}^{-1}$ (Campbell \& Walker 1979; Cochran 1988; Cochran \& Hatzes 1990; McMillan et al. 1990; Marcy et al. 1993; Murdoch et al. 1993). Most of these instruments include an absorption cell in front of the spectrograph (HF or iodine cell) to superimpose absorption lines on the stellar spectrum. That solution prevents errors caused by different optical paths crossed by the stellar beam and the calibration lamp beam. But, with these instruments, only bright stars have been monitored due to the very high signal-to-noise ratio required for high precision Doppler measurements. As a result, only limited samples of stars in the solar vicinity have been measured and no detection of Jupiter-size planets has been reported (Marcy \& Butler 1994; Walker et al. 1995).

The option chosen for ELODIE does not make use of an absorption cell in front of the spectrograph. The problem of the illumination of the entrance slit and collimator is almost completely eliminated by using an optical fibre to feed the spectrograph (Heacox 1986). This also allows us the design of a fixed position spectrograph in an isothermal environment. The condition of identical path for star and calibration is also provided by the high stability and the scrambling effect of the optical fibre. However, long term thermal relaxation of the spectrograph as well as air pressure variations induce non negligible drifts in the spectrograph. An accuracy of a few meters per second is only possible if a simultaneous calibration is done. ELODIE has two fibres (one for the star, the other for the sky). Tests and measurements carried out during the first year of operation have revealed that the relative drift between the sky spectrum and the star spectrum is highly corre- lated. Therefore, we use the sky fibre illuminated by the thorium-argon lamp during the stellar exposure to monitor the spectrograph calibration variation. The results reported in the last section of this paper indicate that a similar accuracy to that of the absorption cell instruments is achieved. To increase the long term stability of ELODIE, a fixed optical system has been chosen, which certainly introduces some limitations for spectroscopy. However, the available wavelength range is wide $(\sim 3000 \AA)$. It allows the simultaneous measurement of calcium $\mathrm{H}$ and $\mathrm{K}$ lines and of the hydrogen $\mathrm{H}_{\alpha}$ line.

While ELODIE has been carefully designed to be able to provide accurate radial velocities on a long time span (Jupiter-size planets and brown dwarf searches) as well as on very short time (asteroseismology), it is also true that this instrument is perfectly suited to the measurement of fairly accurate radial velocities $\left(1 \mathrm{~km} \mathrm{~s}^{-1}\right)$ of very faint stars. Such a domain of application is certainly promising. Cross-correlation spectroscopy also offers an efficient way of measuring rotational velocities of late-spectral type stars (Benz \& Mayor 1981, 1984) and the stellar metallicities of faint stars (Mayor 1980, Pont et al. 1995b). But more can be extracted from spectra if specific templates are designed by using one's a priori knowledge of stellar spectra, a field for future developments with any high resolution echelle spectrograph. For example, the comparison of cross-correlation functions computed with the same spectrum but using different sets of spectral lines of increasing Landé factors can reveal some information on the surface magnetic field of stars (Babel et al. 1995; Queloz et al. 1996).

A crucial aspect of optical cross-correlation instruments is their capacity to provide instant measurements of radial velocity. An important software development has been done to preserve this aspect and to provide a complete on-line reduction of the CCD frames, giving access to the calibrated spectrum and its radial velocity a few minutes after the end of the exposure. The main features of the software are described in this paper.

ELODIE is an Haute-Provence Observatory instrument designed and built in the context of a collaboration between the Haute-Provence, Marseille and Geneva Observatories. A second copy of this spectrograph, called CORALIE, will be located on the new 1.2-meter telescope to be installed at la Silla Observatory (ESO, Chile) by the Geneva Observatory.

A general description of the instrument is presented in the next section. The main features of the reduction software are outlined in Sect. 3. Section 4 is dedicated to the first results and the performances of the instrument. ELODIE as part of the accelerometer instrument designed by Connes (1985), being still under development, is not described here. 


\section{Instrument design}

\subsection{Principle of the "white pupil" mounting}

In an astronomical spectrograph the camera aperture diameter is larger than the disperser size and increases according to the field size. Thus, it is quite impossible to have a high aperture ratio for the camera if a large and very dispersive grating is used.

On the contrary, this limitation does not exist in the "white pupil" mountings: the disperser is re-imaged on the camera and monochromatic beams intersect each other in the new pupil which has to be superimposed on the camera entrance. The magnification between these two pupils (disperser and camera) is a free parameter which has to be chosen in accordance with the camera's optical properties, but the camera size is independent of the disperser size.

In all previously described "white pupil" mountings a more or less complex dioptric system (i.e. a double-pass Littrow collimator) gives, when a reflexion grating is used, a first intermediate spectrum in the vicinity of the slit. In this intermediate space, transfer optics which provide "white pupil" conjugation can either be used as simple field optics, which doesn't change the Littrow aperture, or as a second collimator for another (cross) disperser needing parallel light illumination.

The absence of constraints in the choice of transfer optics leads to a large variety of mountings which share some useful properties:

1. The use of a Littrow system for the reflexion grating is particularly interesting since the angular dispersion is larger. Let us recall here that, for a ruled reflexion grating, the efficiency varies with the cosine ratio of incident and diffraction angles when the mounting deviates from the Littrow conditions.

2. There is no vignetting in the spectrograph.

3 . For a given aperture image, playing with pupil magnification allows the designer to limit field aberrations to the detriment of aperture aberrations and vice-versa (field angle varies with camera size).

\subsection{Optical layout}

The echelle-grating used has a $\tan \theta=4\left(\theta=76^{\circ}\right)$ blaze angle. It is a very impressive optical component $(408 \times$ $102 \mathrm{~mm}$ with $31 \mathrm{~g} \mathrm{~mm}^{-1}$ ) produced by Milton Roy. Using a Tk1024 CCD with $24 \mu \mathrm{m}$ pixels as detector we record, in one single exposure, a spectrum from $3906 \AA$ to 6811 A distributed over 67 orders. The focal length of the camera is adjusted to avoid any gap in this range and to record all the wavelengths even in the extreme red orders. This leads to a focal length of $300 \mathrm{~mm}$, an aperture ratio $f / 3$ and a reciprocal linear dispersion of $2 \AA / \mathrm{mm}$ in the middle of the range $(4800 \AA)$.

The spectrograph is made up of two levels. Used in the Littrow condition but in conic dispersion the echelle grat- ing is put at the common focus of both adjacent mirrors M1 and M2 (see Fig. 1 for a display). It forms the pupil of this Czerny-Turner mounting which constitutes the first level of the spectrograph. An intermediate spectrum is observed in a position symmetrical to the source with respect to the grating. This first spectrum is itself constituted by all the overlapping orders which will be disentangled in the second level.

With a fibre-fed spectrograph, the collimator focal ratio is a free parameter (in so far as adequate transfer optical systems make numerical aperture between fibre and collimator fitted together, see below). We chose $f / 15$ in order to prevent off-axis effects. The intermediate spectrum lies in a telecentric space. It appears rectilinear in the sagittal focal surface which is plane. The spherical aberration is negligible due to the small aperture angle, and there is no coma thanks to the Z Czerny-Turner mounting. In fact, the only aberration we observe is the same astigmatism for all wavelengths coming from the off-axis illumination of M1 and M2.

A small mirror near the intermediate spectrum sends back the monochromatic beams towards a third concave mirror M3 adjacent to the Czerny-Turner mirrors. This third mirror is a new collimator for all these beams which, after the mirror and at its focus, forms a "white pupil" in parallel light. This pupil is the white image of the echellegrating (diameter $102 \mathrm{~mm}$ ) but reduced to a diameter of $75 \mathrm{~mm}$, which is more than enough to obtain a good correction of aberrations. It is worth noticing that the Zmounting between pupils also reduces the pupilar coma.

The dispersion crossing is done by a pair of dispersers, the first being a prism, the second a grism (one deals with the subject as it is currently done for chromatism). All beams cross first a $40^{\circ}$ flint prism, then an $8^{\circ} .63$ crown grism with $150 \mathrm{~g} \mathrm{~mm}^{-1}$. Both dispersers work at minimum deviation. Note that, if the separation of the orders were done only by a prism, we would notice more space between blue orders than between red ones. If, on the contrary, the separation were done by a grism, more space would be observed between the red orders. Combining these two effects, we obtain a constant inter-order spacing over the whole wavelength range, and we record 67 orders on our $1024 \times 1024$ CCD (see Fig. 2).

\subsubsection{Camera}

From the above, one might think that the only aberration which will be observed at the focus of a camera computed for infinity would be off-axis astigmatism. In fact, being the same for all image points, this astigmatism is corrected by an appropriate toroidal deformation on the optical surface very close to the pupil. In ELODIE the object field is very small, and it is therefore easy to make this deformation on the first mirror M1. The camera has therefore 


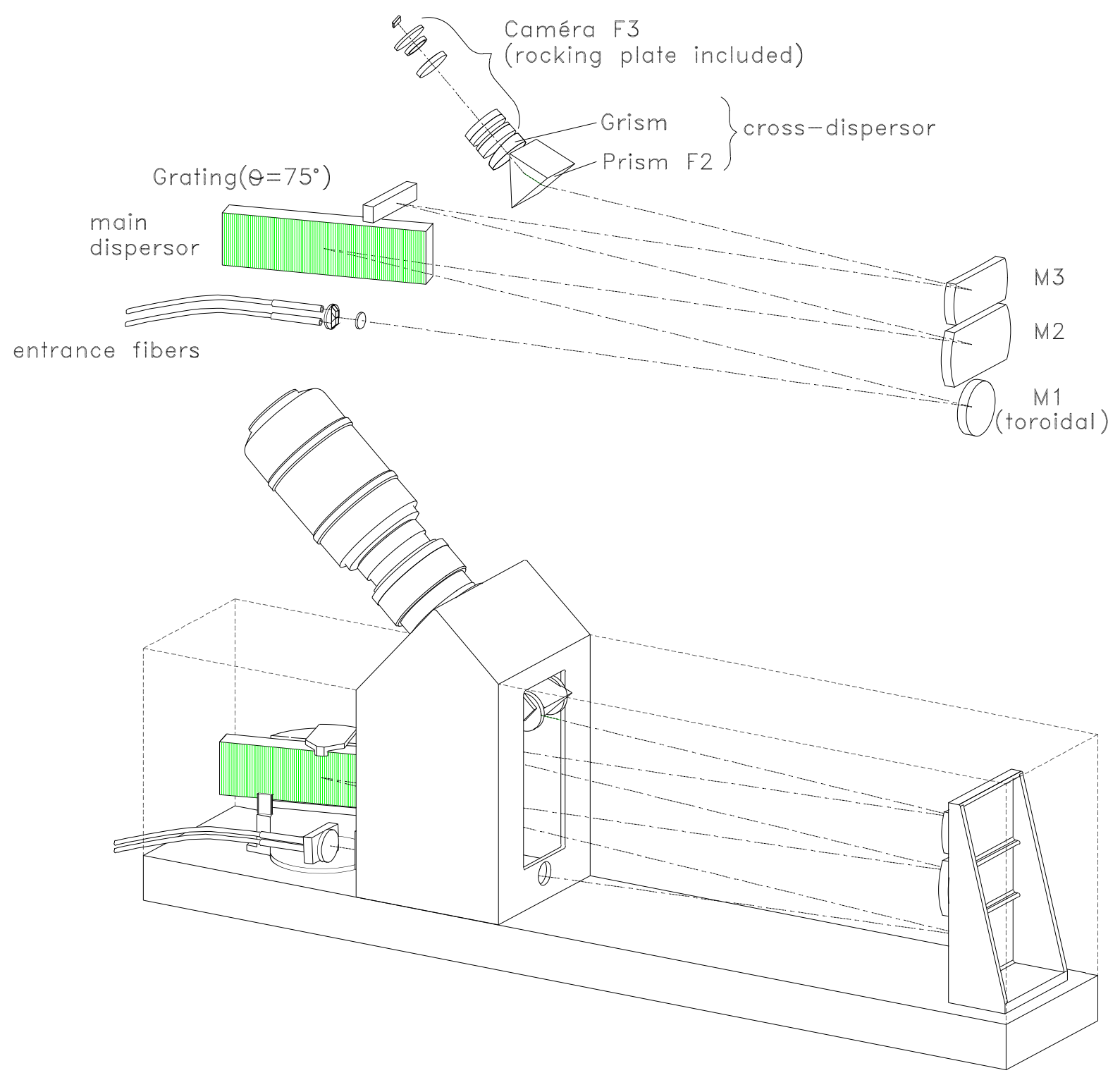

Fig. 1. Optical layout and perspective view of ELODIE

been computed for an aberration-free field at infinity. Its aperture is $f / 3$ with a diameter of $75 \mathrm{~mm}^{1}$.

The small cylinder effect observed in the field, due to the crossing of prisms, is corrected by adjusting the secondary chromatism in the camera. The ELODIE poten-

${ }^{1}$ The camera's real focal length of $225 \mathrm{~mm}$ has to be taken into account in calculating the cross-dispersion, but the pupil magnification affects the calculation of the main dispersion since (as we have seen above) the equivalent focal length is $300 \mathrm{~mm}$. tial resolution is about 100000 (see the spot-diagrams in Fig. 3).

Note that a removable rocking parallel plate (UBK $7 / 20 \mathrm{~mm}$ ) is installed inside the camera in order to move the image without degrading the optical quality of ELODIE. This equipment is part of the accelerometry instrument of Connes. 


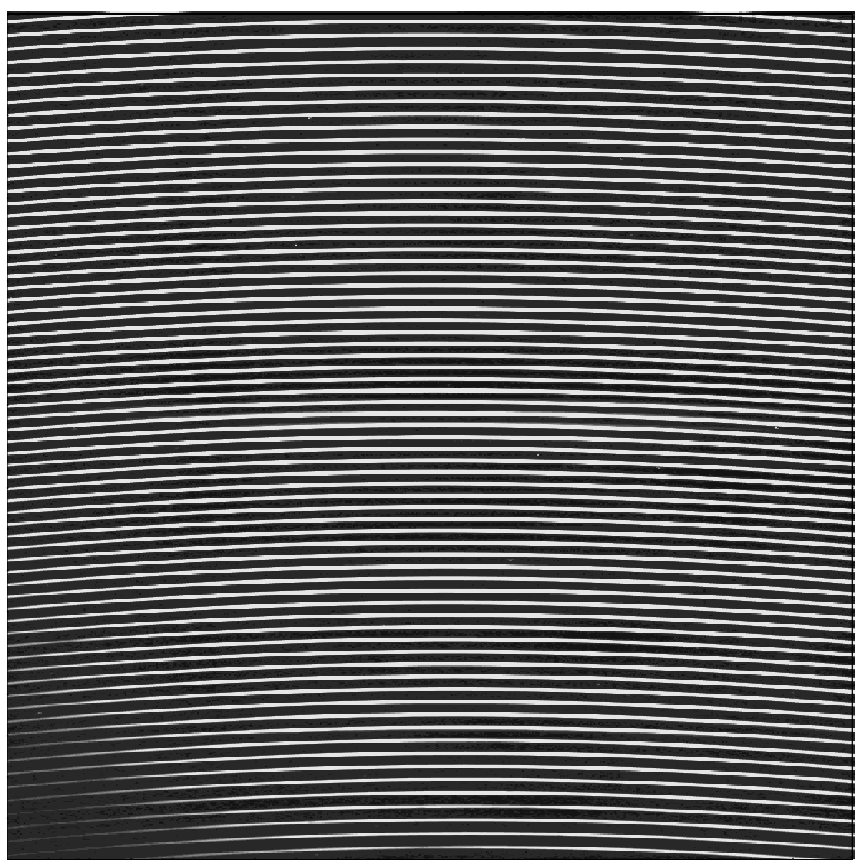

Fig. 2. ELODIE echelle spectrum of a hot star recorded on the CCD Tk1024. 67 orders are usable, starting with the 156th order and ending with the 90th (ranging from $3906 \AA$ to 6811 $\AA$ ). Note that the orders are clearly separated and that the inter-order spacing is remarkably constant over the whole wavelength range

\subsubsection{Parasitic orders and filters}

As we have seen, with a single $1024 \times 1024 \mathrm{CCD}$, a 3000 $\AA$ wavelength range is recorded at one time with a maximum resolution of 100000 . The optical system required to obtain this result is rather complex and has numerous surfaces. The difficulties which this entails must be solved with care.

The first difficulty is a standard problem. The transmission has to be best within the useful wavelength range and reduced as much as possible outside this band. This passband function is obtained by a combination of extralow reflection coatings on the glasses and special dielectric mirrors.

The second difficulty is unusual and more tricky to handle. The cross-dispersion is obtained by two components: the prism and the grism, whose effects are added. The first order of the grism is used, but wavelengths in this order are already widely dispersed by the prism, as well as the wavelengths in orders 0 and 2 . Thus, on the blue part of the CCD appear red wavelengths, while on the red side of the echelle spectrum, the blue wavelengths of order 2 are visible. The rejection of the unwanted orders of the crossdisperser is done by a filter with two coatings inserted in front of the CCD. The first filter, a short-wavelength pass filter, is located on the blue side of the echelle spectrum and the second, a long-wavelength pass filter, is located on

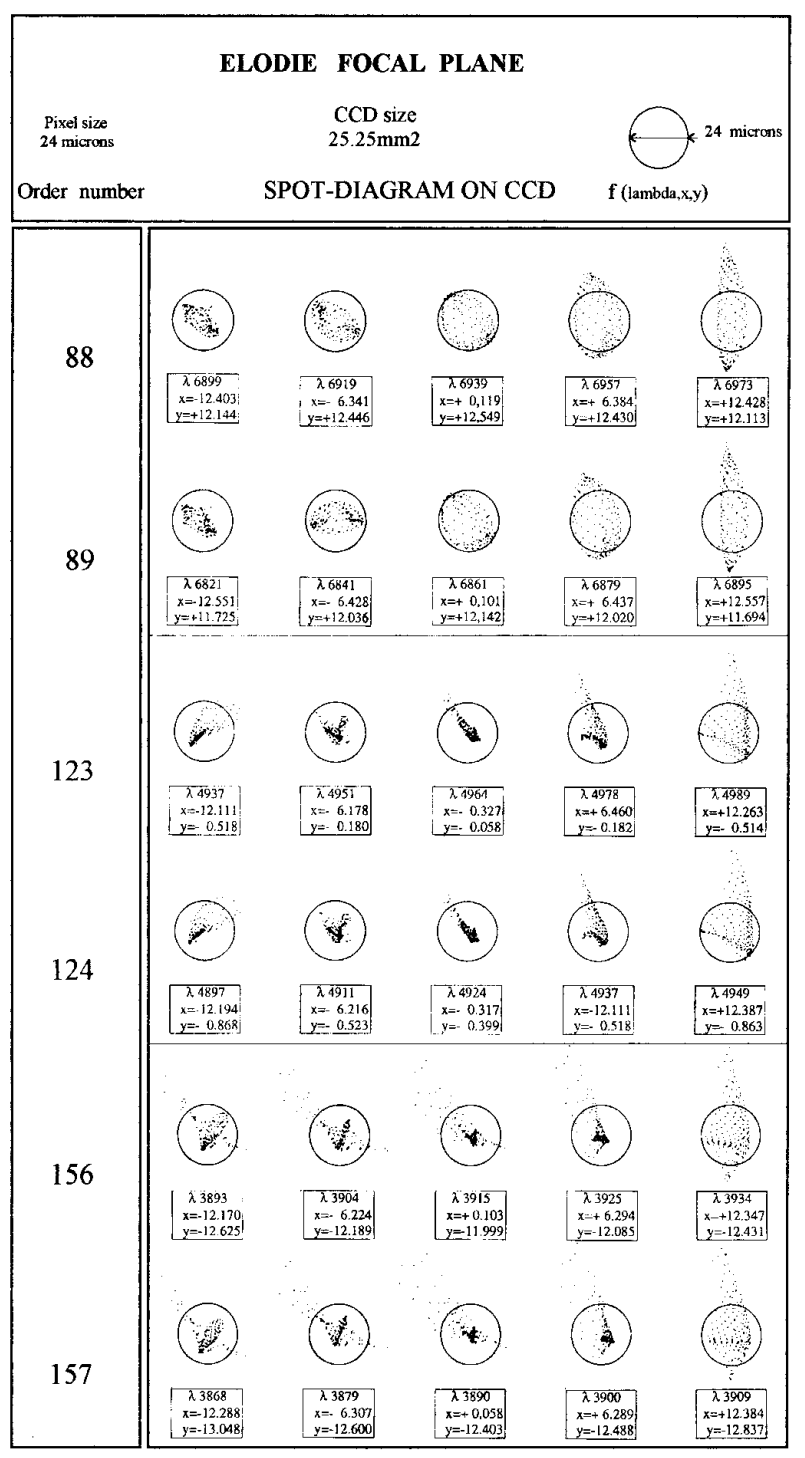

Fig. 3. ELODIE spot-diagrams

the red side. These two additional filters are not adjacent, because the central part of the spectrum is not polluted. As the CCD is much more sensitive in the red, a shortwavelength pass filter is also added at the entrance of the spectrograph. This filter, combined with the dielectric mirrors, increases the efficiency of the short-wavelength pass filter (only below $7000 \AA$ ). It is worth adding that the two sides of these filters, as well as the other diopters of the spectrograph, are also coated with an extra-low reflection coating.

The transmission of the whole useful wavelength range is not particularly influenced by these additional filters. The filters are also located far enough from the CCD to avoid any photometric effect at the limit of the coated areas. However the location of the long-wavelength pass 
coating does not match perfectly all the area contaminated by the wavelengths of the order 2 . Therefore a slight blue contamination remains in the middle of the CCD (see Fig. 11 below)

\subsection{Front-end adaptation to the telescope}

An adaptor, located at the $f / 15$ Cassegrain focus, and two optical fibres provide the optical link between the telescope and the spectrograph, which is kept in an isolated room. The front-end adaptor is composed of many mechanisms. It holds the calibration lamps, does the correction for atmospheric differential refraction and allows the stellar field visualization and the guiding (see Fig. 4).

\subsubsection{Stellar field visualization and guiding}

A tilted steel concave mirror pierced with two holes of $300 \mu \mathrm{m}$ diameter ( $2 \operatorname{arcsec}$ on the sky) allows the fibres to be fed and, at the same time, the image of the field to be reflected onto the guiding camera. These holes are hereafter called: star-hole and sky-hole.

A shutter in front of the camera and a density wheel are also available to protect the guiding camera during the setting of the telescope and the calibration exposures. The automatic guiding is done on the video image.

\subsubsection{Calibration exposures}

The beam coming from the calibration sources is split so as to obtain two distinct optical paths keeping the same pupil. This optical design is highly diffuseless and allows short exposure times. It also prevents the scattered light of the calibration lamp from reaching the guiding camera and the star-hole during a simultaneous exposure on a star (simultaneous thorium).

Several types of calibration are provided by the adaptor, (see also Table 1 for a summary of all the combinations):

1. Illumination by the tungsten lamp to locate the position of the orders and to measure the flat-field. In order to have a good signal over the whole wavelength range, a color corrector filter is inserted to transform the tungsten spectrum, mostly red, into a rather flat spectrum. The fibre mask makes the selection of the hole to be illuminated, in order to locate independently each spectrum.

2. Illumination by the thorium-argon lamp to calibrate the spectra in wavelength.

3. Illumination of the sky-hole by the thorium-argon lamp during a stellar exposure. This observing mode is dedicated to high precision radial velocity measurements. During such exposures, the calibration beam is attenuated according to the exposure duration, in order to get a total illumination by the thorium-argon lamp independent of the exposure time.
4. In the use of ELODIE as part of the accelerometry experiment, the fibres have to be illuminated with the light from a Fabry-Perot interferometer instead of the thorium-argon lamp. In this case, the light from an external Fabry-Perot is carried to the front-end adaptor by means of a third fibre.

Table 1. List of all possible exposure combinations using the calibration lamps

\begin{tabular}{ccc}
\hline calibration type & star-hole & sky-hole \\
\hline 1 & tungsten & tungsten \\
2 & thorium-argon & thorium-argon \\
3 & star & thorium-argon \\
4 & Fabry-Perot & Fabry-Perot \\
4 & star & Fabry-Perot \\
4 & thorium-argon & Fabry-Perot \\
\hline
\end{tabular}

\subsubsection{Atmospheric differential refraction correctors}

This system limits as much as possible the loss of star light by the differential atmospheric refraction at the entrance of the fibre. In term of performances such a system is less demanding than would be, for instance, a speckle image system. However, it must not reduce the size of the field for the setting.

The atmospheric dispersion correction is done with a set of four normal field prisms and a parallel plate. As the coma coming from the use of the convergent beam before the Cassegrain focus of the telescope is negligible, this solution is very convenient and provides good transmission. The choice of the prism set is done so that the secondary spectrum never exceeds 0.3 arcsec in length up to a $\sec z=2$ telescope position.

\subsection{Optical fibre link}

We use POLYMICRO fibres FHP, 20 meters long, with a diameter of $100 \mu \mathrm{m}$ (Avila 1988). Our choice is to form the star image and not the pupil on the fibre entrance. Note that it would be interesting to compare the quality of both solutions in term of efficiency and radial velocity accuracy. The beam apertures are converted to $f / 5$ with a transfer optic to feed the fibres and then brought back to $f / 15$ at the spectrograph entrance. In the end, at the focus of the $f / 3$ camera lens, the geometrical spot diameter is $60 \mu \mathrm{m}$. Therefore the effective resolution of ELODIE is about 40000 .

The main difficulty in the fibre mounting comes from the optic cable SMA connectors. The final quality remains strongly dependent on the overall assembly. We have designed a device to control the conservation of the inputoutput numerical aperture of fibres. The method consists 


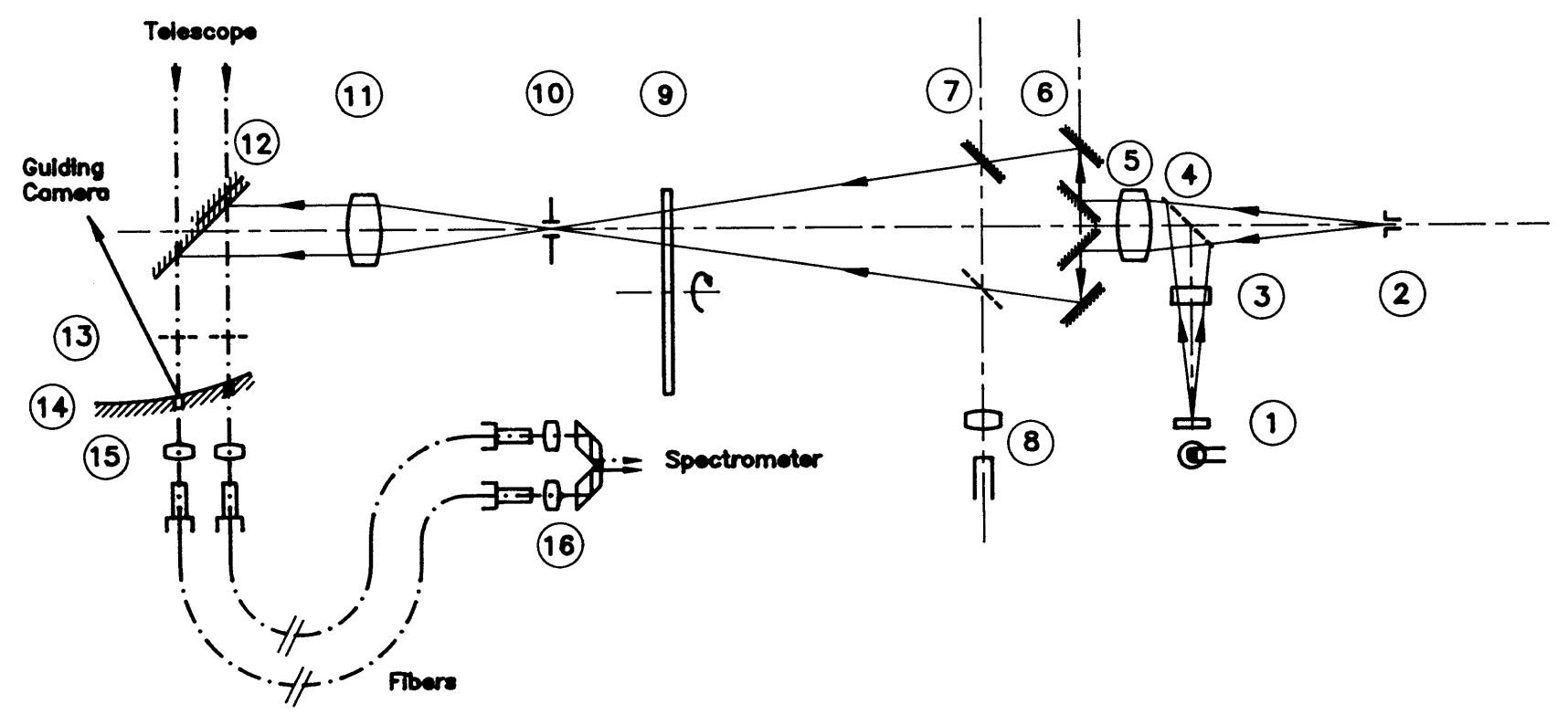

Fig. 4. Optical layout of the calibration system in the front end adaptor: 1) tungsten lamp and diffuser, 2) hollow cathode thorium lamp, 3) colour corrector filter. 4) static beam-splitter, 5) relay achromat, 6) mirrors for beam angular splitting, 7) removal mirror and beam splitting (part of the accelerometry experiment), 8) optical fibre of the Fabry-Perot light (part of the accelerometry experiment), 9) circular wedge 10) pupil, 11) achromat (to put the pupil at infinity), 12) calibration mirror, 13) fibre mask, 14) concave mirror with 2 holes, 15) spectrometer linking fibres and aperture converter, 16) aperture converter optics and rhomboid prisms (to bring the beams closer to the focus of the collimator)

in illuminating the entrance of the fibre with a beam of fixed aperture (here $f / 4.5$ ) and to analyse the flux at the end of the fibre through a diaphragm of variable aperture. In Fig. 5 we see that it is possible to obtain a final transmission close to the optimum, limited only by the loss in the glass and the air-glass interface.

\subsection{Exposure meter}

An exposure meter records continuously the flux which enters the spectrograph. This information is very helpful in selecting the optimum observing time. Furthermore, this allows us to compute the true mean time of the exposures, which is critical for accurate correction for the Earth's velocity. Note that the variation of the radial projection of the Earth's motion on the line of sight, at OHP, can be as large as $1.5 \mathrm{~m} \mathrm{~s}^{-1} \mathrm{~min}^{-1}$.

The exposure meter is fed by the light out of the efficient beam, as near as possible to the blaze. A spherical mirror (aperture $f / 1$ ) located on the normal side of the grating and a bundle of optical fibres lead the light to a photomultiplier outside the spectrometer. We have measured that $3 \%$ of the light entering the spectrometer reaches the photomultiplier. It is worth noting that we tried another method consisting in collecting the light of low and grazing orders but only collected $0.3 \%$ of the incoming flux.

\subsection{Detector}

A back-side illuminated Tk1024 with $24 \mu \mathrm{m}$ pixels is used. It is cooled to $183 \mathrm{~K}$. Particular attention is given to the temperature regulation in order to insure good stability. At this temperature, using a slow read out $(100 \mu \mathrm{s} /$ pixel $)$, we measure a typical read-out noise of $8.5 \mathrm{e}^{-}$. The CCD quality is typical of a grade 1 and its linear response is excellent up to $100000 \mathrm{e}^{-}$. Four gains are available: 4, 2, 0.8 and $0.4 \mathrm{e}^{-} / \mathrm{ADU}$.

\subsection{Instrument control}

Five computers are used to control the instrument:

- a workstation to control the observations.

- a workstation reserved for the on-line reduction.

- a PC to control the specific electronic interface, which carries the opto-electronic isolation and the signal adaptation, to control the different motions and to display the status.

- a CCD controller 


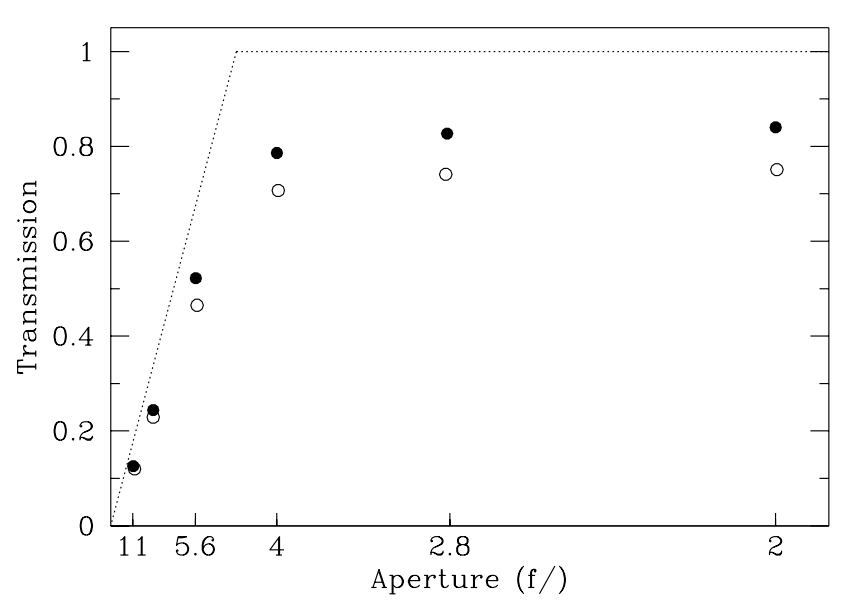

Fig. 5. Transmission of the fibres for various output apertures. The entrance beam has a fixed aperture of $f / 4.5$. The dotted lines represent the incoming flux. The filled and empty dots indicate the flux coming out at respectively $\lambda=5461 \AA$ and $\lambda=4047 \AA$

- an HP1000 computer to control the telescope and to give its position.

All these computers are connected via an Ethernet network. They communicate using the client-server protocol.

The use of ELODIE is fully automatic, no manual action is needed to configure the spectrograph and carry out the observations. A specific user interface is used by the observer to start the exposures. All the actions are then automatically carry out up to the end of the data reduction. The "pipeline" includes the exposure configuration, the exposure itself, the data acquisition and the reduction. The stellar exposures are defined by their exposure times but, thanks to the exposure meter, a signal-to-noise ratio to be achieved can also be requested.

The sequence of exposures is treated simultaneously, as a stack, along the pipeline in order to spare as much time as possible. The maximum stack available by the system is the following: an exposure in the reduction program; an exposure waiting for reduction; the exposure in progress and an exposure defined, ready to be started.

\section{Data reduction}

The use of fibres to feed the spectrograph makes the position and the shape of the orders stable on the CCD and independent of the seeing. Therefore, it is possible to define an optimum data reduction procedure and to apply this treatment algorithm, in an automatic way, for all the spectra observed. Such a program, specifically adapted to the instrument, provides a set of homogeneous spectra and saves the observer the labour of reducing the echelle spectra.
An automatic on-line data treatment, completely integrated in the spectrograph operation train, is available with ELODIE. The images are reduced directly after the exposures. Considering the quality of the algorithms used, the ELODIE reduction must not be seen as a quick look facility but as an extension of the spectrograph abilities, providing reduced data instead of "classical" CCD raw images.

The work itself is done with an interpreter, dedicated to the fast treatment of astronomical images (Weber 1996, in preparation). A set of specific algorithms has been developed in this environment to reduce echelle spectra.

\subsection{Overview of the reduction procedure}

All the images coming from the spectrograph are saved on a disk for security purposes. The classical treatment of CCD frames is then carried out: correction of bad pixels, offset and dark current subtraction. Note that, as the offset and the dark are not constant over the CCD, averages of $64 \times 64$ pixels are used, for both images, instead of a single average value.

The determination of the position of the orders is achieved by illuminating the fibre entrances with the tungsten lamp. The geometrical definition of the sky orders and star orders are carried out in two distinct exposures. From these images, the orders are geometrically characterized and the blaze function and the flat-field spectrum are also computed.

The wavelength calibration is done with the thorium lamp. After the extraction of the orders, the thorium emission lines are identified on the spectrum and the wavelength geometry is fitted using a two-dimensional polynomial expansion of the Littrow approximation for the echelle grating solution. The quality of the solution is finally controlled by computing the cross-correlation function of the thorium spectrum. The focus of the spectrograph is also checked during this operation.

A "true" flat-field image with widened orders is available with ELODIE. The width of the orders is artificially increased by changing the position of the image of the fibre entrance in the spectrograph. This image, called flat-field image $^{2}$, gives after some treatment the relative response of the pixels in the direction perpendicular to the dispersion $(Y$-direction) for each order and at each resolution bin along the main dispersion ( $X$-pixels). The response of the pixels along the main dispersion is given by the flat-field spectrum. It is computed from the extracted spectrum of a tungsten exposure (not broadened) previously divided by the flat-field image (before the extraction). For all the stellar exposures, the flat-field correction is then made in two steps: first dividing the image by the flat-field image and then the extracted spectrum by the flat-field spectrum.

\footnotetext{
${ }^{2}$ not to be confused with the flat-field spectrum.
} 

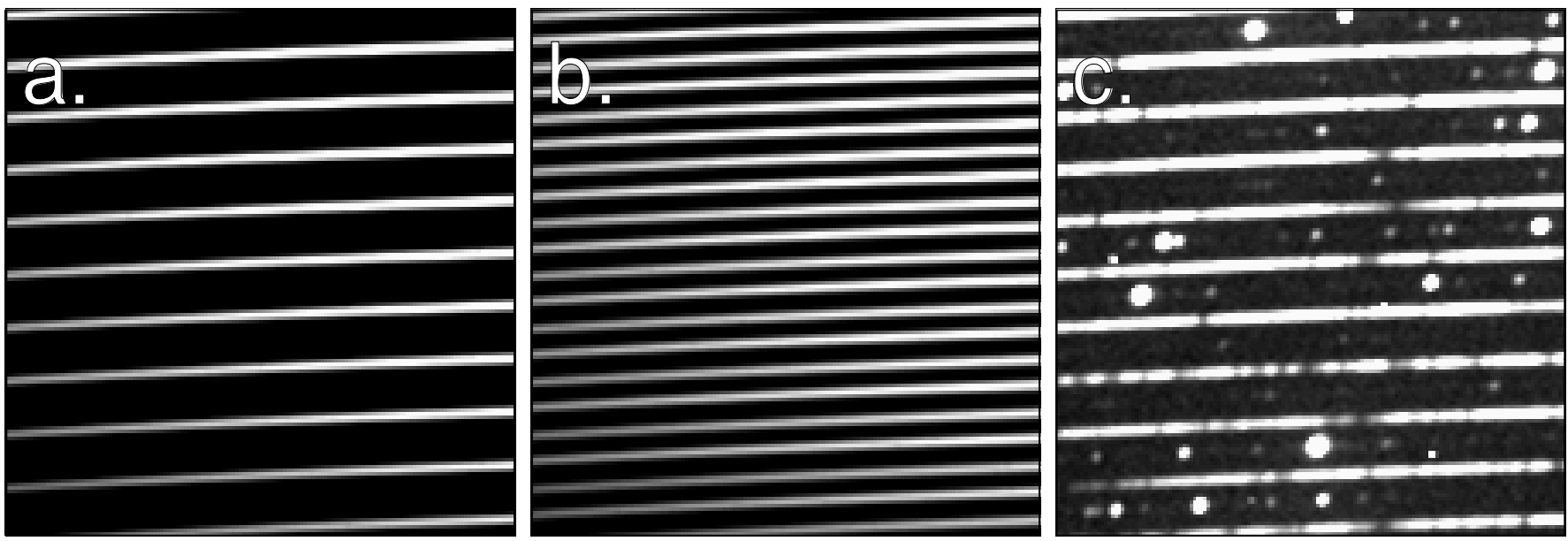

Fig. 6. Small part of the raw image for the three different types of stellar exposure available. a) "star fibre only", b) star fibre and sky fibre simultaneously illuminated (here by the tungsten lamp), c) "simultaneous thorium": star on the star hole and the sky hole simultaneously illuminated by the thorium lamp

Note that this correction in two steps is not necessary for spectra with $S / N<300$.

Three stellar exposure types are available (see Fig. 6): (1) "star fibre only": the fibre mask is set on the sky-hole; (2) "star fibre and sky fibre": the fibre mask is out of the telescope beam in order to measure the sky spectrum as well; (3) "simultaneous thorium": during the exposure, the thorium lamp illuminates the sky fibre to monitor the spectrograph drift.

The observations of relatively bright stars, requiring careful spectroscopic reduction, is done with "star fibre only" exposures. The inter-order spacing is large enough accurately to estimate the background contamination and to correct for it. In the case of faint objects, which can be significantly contaminated by the skylight, a "star fibre and sky fibre" exposure is to be preferred. For such an image however, as the orders are too close, no background subtraction is possible. For both exposure types, the orders are then extracted and wavelength calibrated using the last thorium exposure carried out.

For the simultaneous thorium exposure, the thorium spectrum is extracted, calibrated with the last thorium exposure and then cross-correlated. The wavelength solutions of each way (sky and star) are independent but their drifts are similar (see part 4.1). Therefore, the velocity shift of the sky spectrum corresponds to the measurement of the mean spectrograph drift relative to the last wavelength calibration. As for the "star fibre and sky fibre" exposure there is no background subtraction.

For all the exposure types, the observer has the possibility of computing cross-correlation functions. The algorithm uses "binary" templates and mimics the CORAVEL process (e.g. Baranne et al. 1979; Queloz 1995b).

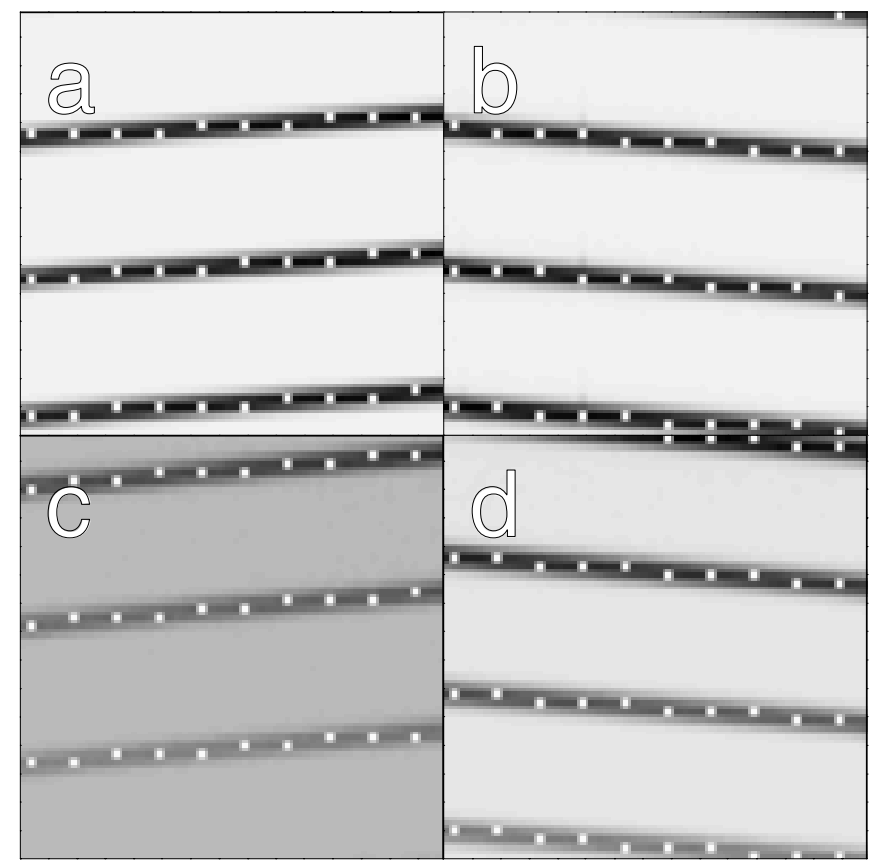

Fig. 7. Display of the location of the orders found by the program (white points), in the four corners of the image: a) top left, b) top right, c) bottom left, d) bottom right. Note that the display of the position of the orders is here sampled by the CCD. Obviously the position is known to a better accuracy than the pixel size

\subsection{Geometrical definition of the orders}

The geometrical definition of the orders starts with the search for the order location in the middle of the image along a column (in the $Y$-direction). Then, the algorithm computes the centre of gravity $y_{c}$ of each order at every $x$ position, moving step by step from the 


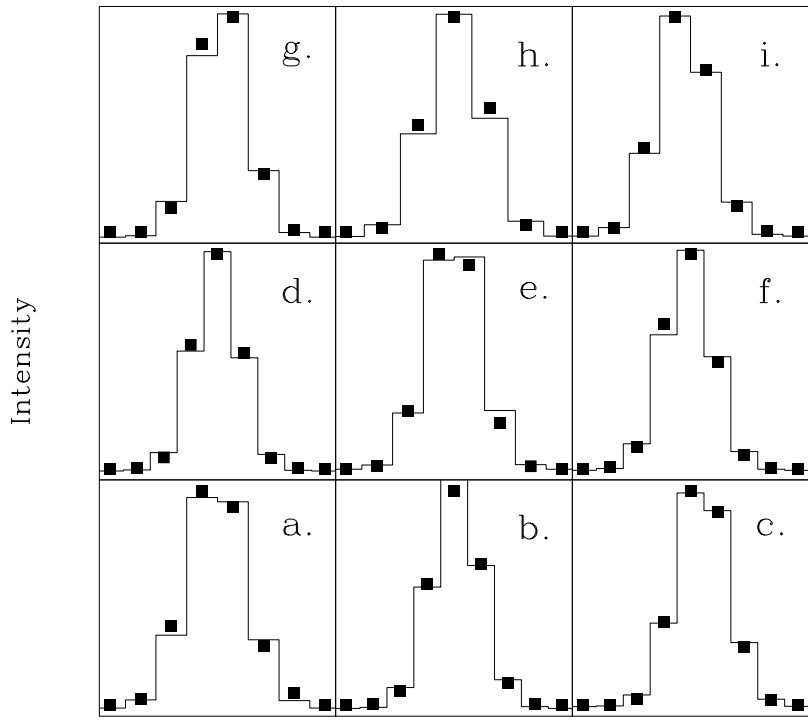

Y pixel

Fig. 8. Profiles of the orders for a localization image (black squares) compared to the fitted Gaussian shape for various positions on the image. The profiles from a) to c) correspond to the 152 nd order for the $X$-position 79,487 and 946 respectively. The profiles d) to f) are for the 124 th order and $\mathbf{g}$ ) to i) for the 94 th order

centre to the edges of the image. Finally, a two dimensional polynomial is fitted to constrain the order location: $y_{\mathrm{c}}(o, x)=\sum_{i, j} a_{i, j} o^{i} x^{j}$, where $o$ is the order number (internal numbering) and $x$ the pixel position. To get a good solution out to the edges of the image, a high signal-tonoise ratio spectrum is used and the image background is carefully subtracted. In Fig. 7, the position of the orders found by the algorithm, in the four corners of the image, is displayed.

The shape of the orders perpendicular to the dispersion (spatial profile) is approximated by a Gaussian. To fit this shape throughout the image, a set of 2-dimensional functions $G_{i, j}^{\mathrm{e}}(x, y)$ (called herein after elongated Gaussians) is used:

$$
G_{i, j}^{\mathrm{e}}(x, y)=A_{i, j} \exp \left(-0.5 \frac{\left(y-y_{\mathrm{c}}(x, o)\right)^{2}}{\sigma_{i, j}^{2}}\right) .
$$

Each of these functions is fitted over a range of $30 X-$ pixels with the width $(\sigma)$ and the amplitude $(A)$ as free parameters. The previous determination of the position of orders $\left(y_{\mathrm{c}}(x, o)\right)$ is used. The measured $\sigma_{i, j}$ are then fitted with a two-dimensional polynomial to give a continuous description $\sigma(x, o)$ of the width of the orders throughout the image. This algorithm provides a good description of the spatial profile, independent of the order position. The use of elongated Gaussians in the $X$ direction instead of

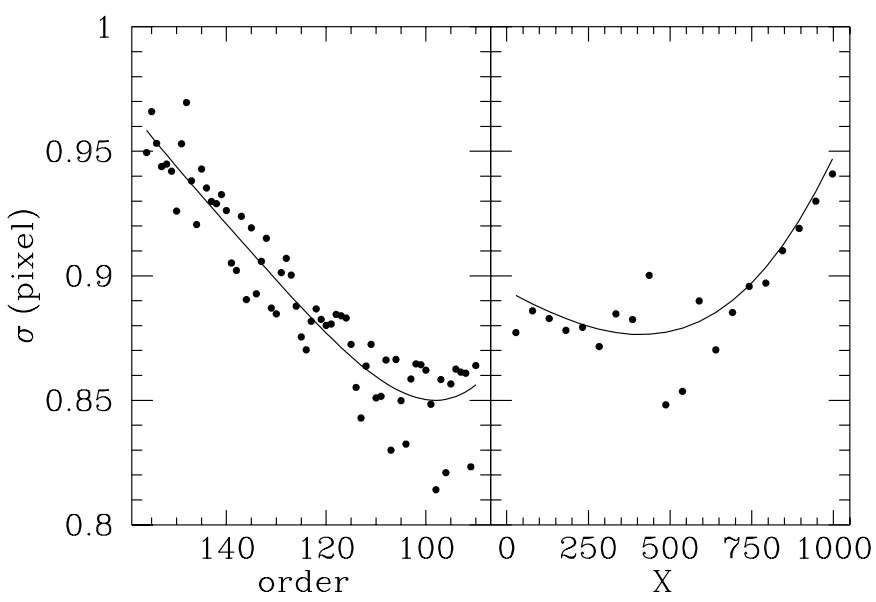

Fig. 9. $\sigma_{i, j}$ measured by the fit of a set of elongated two-dimensional Gaussians (points): left for $X=640$ along all the orders. Right for the central order (124th) along the direction of the main dispersion. The lines are the fitted polynomials $\sigma(x, o)$. The noise around the fitted function is mainly due to the sampling noise

several Gaussians strongly decreases the pixel sampling noise and increases the quality of the measurement. This is particularly important for ELODIE which has narrow and very slightly tilted orders. Figure 8 displays the comparison between the observed profile and the fitted model. One clearly sees that a Gaussian shape is a good approximation to the spatial profile for a fibre-fed spectrograph.

The comparison between the true profile and its adjustment gives a good estimate of the error in the position of the orders found by the program. As the centre is not a free parameter during the fit of the elongated Gaussian, any mismatch would have a direct consequence on the quality of the fit. After some investigations we concluded than if any systematic error greater than 1/10 pixel were present it would be clearly visible.

The variation of the width of the orders across the image, displayed in Fig. 9, shows that the focus perpendicular to the main dispersion is sharper in the red part than in the blue part of the image and that there is a slight effect along each order. The dispersion around the fitted polynomial is mainly due to the sampling effect, particularly at the centre of the image where the orders are almost flat. It is worth noticing that the ELODIE orders are particularly narrow, $90 \%$ of the energy being spread over 3 pixels.

\subsection{Background contamination}

The background emission can be split into two components: a local one due to the inter-order scattering, proportional to the flux in the adjacent orders, and a diffuse 
Fig. 10. a) $S / N$ distribution across the orders for a tungsten spectrum at the location $X=500$ (solid line) and $X=200$ (dotted line). b) the flux ratio (in percent) between the inter-order "spectrum" (or background "spectrum") and the tungsten spectrum. In the red part, the background level is proportional to the flux in the order which is a characteristic of a local background contamination. The rise of the relative background level in the blue part of the spectrum, mirror image of the flux distribution, is a feature of a high contamination by the diffuse component

one, proportional to the overall flux level on the image. Thus the level of the background contamination in each order is proportional to the flux in the order itself but also depends on the ratio between the flux in the order and the level of the overall illumination. In Fig. 10 this two-component effect is clearly visible.

In "star fibre only" exposures, the order separation is large enough to estimate the background emission from the measurement of the flux in the inter-orders. The typical error in the background measurement, using a two dimensional polynomial fit, is about 5\% (see Fig. 11). Taking into account the background level (Fig. 10) we then conclude that, after subtraction, the remaining uncorrected background is lower than $0.5 \%$ of the flux in the order for the great majority of the orders.

\subsection{Extraction of the orders}

The extraction of the orders is carried out using an optimal extraction algorithm with a cosmic-ray hit elimination based on the Horn (1986) algorithm. The spatial profile, previously measured, is used to determine the extraction zone, to compute the weight of each pixel and to detect and correct the pixels hit by cosmic rays. The extraction algorithm can be split into 9 stages:

1. The numerical extraction slit is defined as a multiple of the $\sigma$ of the spatial profile. ELODIE uses a numerical
Fig. 11. Top cross orders tracing at $X=500$ of a localization exposure superimposed with the polynomial fit of the background. We notice around $Y=500$ a little bump due to the contamination by some uncorrected blue wavelength coming from the second order of the cross-disperser. Bottom difference between the fit and the background level $\Delta f / f=\left(f_{\text {image }}-f_{\text {fit }}\right) / f_{\text {fit }}$. The typical error in the background measurements is below $5 \%$

slit of $\pm 3 \sigma$ leading to the extraction of $99.7 \%$ of the flux spread over the orders.

2. A first estimate of the extracted spectra $E_{x}^{0}$ is computed: $E_{x}^{0}=\sum_{y} I_{x, y} b_{x, y} M_{x, y}$, where $b_{x, y}$ is the real fraction of the pixel to extract, $I_{x, y}$ the flux in the pixel $x, y$ and $M_{x, y}$ the mask for the cosmic ray hits (at the beginning $M=1, \forall x, y)$.

3. The expected profile $p_{x, y}$ is computed and sampled in "real" pixels. The following normalization is then enforced $P_{x, y}=p_{x, y} / \sum_{y} p_{x, y} M_{x, y}$.

4. The variance image is computed: $V_{x, y}=P_{x, y} E_{x}^{0}+\sigma^{2}$, where $\sigma$ is the read-out noise of the CCD per pixel.

5 . The spectrum is extracted using the optimal extraction equation of Horn:

$$
E_{x}=\frac{\sum_{y} M_{x, y} P_{x, y} b_{x, y} I_{x, y} V_{x, y}^{-1}}{\sum_{y} P_{x, y}^{2} V_{x, y}^{-1}} .
$$

6. A Search for any cosmic ray hit is done using the Horn criterion: $\left(b_{x, y} I_{x, y}-E_{x} P_{x, y}\right)^{2}>\sigma_{\text {clip }}^{2} V_{x, y}$ with $\sigma_{\text {clip }}=$ 4.5 and a parameter $X_{\max }$ to take into account any error in the profile modelling:

$$
\frac{b_{x, y} I_{x, y}-E_{x} P_{x, y}}{b_{x, y} I_{x, y}+E_{x} P_{x, y}}>X_{\max } .
$$


When both conditions are true, the cosmic ray is detected.

$X$ max is defined for 4 parameters: (1) $\varepsilon_{\mathrm{c}}$ (in pixel) to describe a centering error, (2) $\varepsilon_{\mathrm{s}}$ for the error in the width of the spatial profile, as a fraction of $\sigma$, (3) $\varepsilon_{\mathrm{b}}$ to take into account any uncorrected background level, as a fraction of the amplitude of the profile and (4) $\varepsilon_{\mathrm{a}}$ to take into account any uncorrected relative variation of pixel sensitivity. It is worth noting that without the addition of such criteria to the "classical" Horn technique, the algorithm would not work on high signal-to-noise spectra without using unrealistic $\sigma_{\text {clip }}$ values. The definition of the spatial profile is never good enough to be used on high signal-to-noise ratio spectra. Using a Gaussian profile, the maximal $X$ values are computed for each parameter:

$$
\begin{aligned}
X_{\max }\left(\varepsilon_{\mathrm{c}}\right) & =\frac{\exp \left(-\Delta y \sigma^{-2} \varepsilon_{\mathrm{c}}\right)-1}{\exp \left(-\Delta y \sigma^{-2} \varepsilon_{\mathrm{c}}\right)+1} \\
X_{\max }\left(\varepsilon_{\mathrm{s}}\right) & =\frac{\exp \left(-.5 \Delta y^{2} \sigma^{-2}\right)^{\frac{2 \varepsilon_{\mathrm{s}}}{1-2 \varepsilon_{\mathrm{s}}}}-1}{\exp \left(-.5 \Delta y^{2} \sigma^{-2}\right)^{\frac{2 \varepsilon_{\mathrm{s}}}{1-2 \varepsilon_{\mathrm{s}}}}+1}, \\
X_{\max }\left(\varepsilon_{\mathrm{b}}\right) & =\frac{1}{1+2 \varepsilon_{\mathrm{b}}^{-1} \exp \left(-.5 \frac{\Delta y^{2}}{\sigma^{2}}\right)}, \\
X_{\max }\left(\varepsilon_{\mathrm{a}}\right) & =\varepsilon_{\mathrm{a}} / 2
\end{aligned}
$$

where $\Delta y$ represents the distance (in pixels) from the profile centre and $\sigma$ the standard deviation of the Gaussian profile in pixels. In the reduction, the following values are used: $\varepsilon_{\mathrm{c}}=0.3$ pixels, $\varepsilon_{\mathrm{s}}=3 \%, \varepsilon_{\mathrm{b}}=4 \%$ and $\varepsilon_{\mathrm{a}}=10 \%$.

7. When the algorithm detects a cosmic ray hit at the position $x_{i}, y_{j}$ it sets $M_{x_{i}, y_{j}} \equiv 0$ and goes back to point (2). This loop is iterated until there is no more cosmic ray detection. The rejection scheme only permits to reject one pixel per iteration. If more than one cosmic ray is detected during one loop, only the pixel with the highest difference compared to the expected value is considered as a hit.

8. The value of hit pixels is estimated and corrected using the remaining information from the "good pixels" and the spatial profile shape.

9. The final extraction is carried out using the algorithm described in point (5) $\left(M_{x, y}=1, \forall x, y\right)$

A slight mismatch between the true spatial profile and the model has no significant influence on the flux extracted by the weighted algorithm. A global vertical shift of about 0.2 pixels or a error of $5 \%$ in the width of the spatial profile adds a "noise" of less than $0.2 \%$ in the extracted flux (see in Fig. 12). This effect is negligible in our case. The systematic errors in the measurement of the width, the location of the orders and the maximal variation of the order positions during the night are lower than these values.
Fig. 12. Simulation of the variation of the extracted flux for various simulated mismatches between the model and the true geometry for the 117 th order $\left(\Delta f / f=\left(f_{\text {true }}-f_{\text {mismatch }}\right) / f_{\text {true }}\right)$. Upper figure the order are vertically shifted of 0.2 pixels. Lower figure the width of the spatial profile is increase by $5 \%$

The basic format of the extracted spectra is twodimensional images, call herein after $2-\mathrm{D}$ spectra. This data structure keeps the original pixel identity along the direction of main dispersion as well as the order number. The wavelength solution is described with polynomial coefficients and stored in the image descriptors. Such a data format offers the possibility of keeping the information on the location of each spectrum bin on the CCD and therefore the original sampling.

\subsection{Flat-field correction}

To correct for the relative pixel sensitivity variation of the CCD, the flat-field exposure must ideally illuminate uniformly the order profile (along the spatial profile). Unfortunately, with a fibre-fed spectrograph, this operation is not feasible because the width of the order is always constrained by the fibre diameter. The standard flat-field correction for these spectrographs is to build a flat-field spectrum and then to divide each order of the star spectrum by the corresponding order of the flat-field spectrum (e.g. Hall 1994). However this procedure is not formally correct: $\sum_{y} I_{x, y} / \sum_{y} F_{x, y} \neq \sum_{y}\left(I_{x, y} / F_{x, y}\right)$ and produces a slight loss of accuracy. It is worth noticing that, if the position and the spatial profile of the orders $P$ remains identical during the exposures of the star and the flatfield spectrum, then $F_{x, y}=P_{x, y} \cdot F_{x}$ and $I_{x, y}=P_{x, y} \cdot I_{x}$. 
Fig. 13. Top Cross orders tracing at $X=500$ of a flat-field exposure, superimposed with a localization exposure for comparison. Bottom displays the same crosscut of a flat-field image (corrected from the non-uniform illumination and normalized)

Therefore, the inequality becomes an equality and the standard flat-field correction is correct. However, this condition is never strictly met despite the high stability of the orders due to the use of fibres.

ELODIE is able to increase the width of the orders by moving the fibre entrance in the spectrograph. Therefore, using the tungsten lamp, we can define a true flat-field exposure. However, the illumination created by the fibre movement is not uniform along the profile (see Fig. 13). To correct for this, the spatial profile of the flat-field, for every resolution bin is normalized and fitted on the extraction domain (in the $Y$ direction) by a second order polynomial. As we expect a global and smooth variation of illumination throughout the image, the variation of the coefficients of the second-order polynomials are also constrained by a two-dimensional polynomial: $c_{k}(x, o)=\sum_{i, j} a_{i, j, k} x^{i} o^{j}$, where $c_{k}$ is the $k^{\text {th }}$ coefficient of the second-order polynomials, $x$ the $X$-pixel position and $o$ the order number. Finally, the normalized flat-field image is divided by the fitted model. In the remainder of this paper, we will consider the flat-field image as the normalised and corrected one.

The correction for the flat-field is split into two operations. First, the image is divided by the flat-field image to correct the relative pixel sensitivity along the spatial profile. Secondly, the $2-\mathrm{D}$ spectrum is divided by the flat-field spectrum, to correct the "extracted pixel". This two-step process permits a formally correct flat-field correction and also provides the possibility of using other
2-D spectra to correct the flat-field in the $X$-direction (a spectro-photometric star, for example). This flexibility is important for an observational system with an automatic reduction procedure where the observer is no longer supposed to work with the raw images. Note that the use of a flat-field image is optional. If there is no flat-field image, only the "standard flat-field correction" is carried out. In particular, for spectra with $S / N<300$ the stability of ELODIE is so good that it is not worth using the flatfield image. Using the standard flat-field correction, with the flat-field spectrum alone, is enough to have a good flat-field correction.

The flat-field spectrum is measured with a localization exposure and reduced in the same way as an astronomical image (offset, flat-fielded with the flat-field image - if exists-, background corrected). Every order is then fitted by a polynomial to model the blaze response and any remaining low frequency component. The flat-field spectrum is finally computed by dividing the localization spectrum by this model.

\subsection{Wavelength calibration}

The wavelength calibration is carried out with the thorium spectrum. The algorithm searches, in the extracted spectrum, for a sample of unblended and relatively strong lines selected from the atlas of Palmer \& Engleman (1983). A Gaussian is then fitted to each detected line to find its position, and a two-dimensional polynomial is used to constrain the global wavelength solution. Finally, the difference between the fit and the location of the lines is computed. The lines with deviations greater than 2.75 times the rms are rejected. This last step is iterated until there is no more rejection. Usually the convergence occurs after about 10 steps and $10 \%$ of the lines of the initial sample are rejected. The polynomial used is an expansion of the grating equation for small deviation angles: $m \lambda=P(x, m)$, where $m$ is the order number and $x$ the pixel position along the direction of the main dispersion. The quality of the calibration is finally checked by crosscorrelating the calibrated spectrum with a thorium template built from the atlas. Every thorium lamp exposure produces a new wavelength calibration which is valid until the exposure of the next one.

The degree of the polynomial is 3 and 5 respectively for the $x$ and $m$ space. The final solution uses typically 1100 lines and the rms between the fit and the location of the lines is always around $0.002 \AA$ (about $120 \mathrm{~m} \mathrm{~s}^{-1}$ ).

To estimate the accuracy of the wavelength calibration, we have computed the cross-correlation function of each order alone for 15 thorium spectra measured over two nights. Within each order, the thorium spectrum has only about 30 lines usable for the cross-correlation. Therefore their radial velocity is not strictly zero because of line mismatching and sampling effects. Nevertheless, the velocity offset of each order must be the same for all the thorium 
Fig. 14. Dispersion of the radial velocity in each order of a thorium spectrum, computed with 15 spectra measured over 2 days. Across most of the spectrum the typical accuracy per order is about $20 \mathrm{~m} \mathrm{~s}^{-1}$

spectra and the velocity dispersion around this value gives the typical wavelength calibration uncertainty for each order. We measure about $20 \mathrm{~m} \mathrm{~s}^{-1}$ (see Fig. 14) typical accuracy per order and estimate the global uncertainty in the radial velocity due to the wavelength calibration alone at about $3 \mathrm{~m} \mathrm{~s}^{-1}\left(\sqrt{\sum \sigma_{i}^{2} / \sum i}\right)$.

\subsection{Computation of the cross-correlation function}

The computation algorithm for the cross-correlation function $(\mathrm{CCF})$ is very much like the optical cross-correlation process used by CORAVEL (Baranne et al. 1979; Queloz 1995b). The template spectrum is made of box-shaped emission lines of equal amplitude and plays the role of the real spatial filter of the CORAVEL mask.

The CCF is computed from a wavelength calibrated $2-\mathrm{D}$ spectrum. For stellar spectra, only the orders having an average signal-to-noise $(S / N)$ ratio greater than 0.15 are used. Monte-Carlo simulations have shown that below this value the orders add noise to the CCF. The value of the Earth barycentric velocity towards the star is taken into account to constrain the wavelength domain of each order so as to keep the wavelength range used in the CCF calculation independent of the period of measurement. From the wavelength limits of each order and the velocity range to compute, the algorithm selects, for each order, the lines which will always be in the wavelength domain during the scan. The CCF $C(v)$ is computed step by step for each velocity point without either rebinning or merging the orders:

$$
C(v)=\sum_{l} \sum_{x, o} p_{l, x, o}(v) f_{x, o}
$$

where $f_{x, o}$ is the value of the $2-\mathrm{D}$ spectrum for the order $o$ at the pixel location $x$ and $p_{l, x, o}$ is the fraction of the $l^{\text {th }}$ line of the template which falls into the pixel $(x, o)$ at the velocity $v$. The CCF is then normalized and brought to the solar system barycentric reference frame using the algorithm of Stumpff (1980). Finally, a Gaussian, or a double Gaussian in case of SB2 spectra, can be fit by the observer.

A systematic study have been carried out in order to avoid any error in the correction of the Earth's motion due to the algorithm itself. In particular we have compared the results from the Stumpff algorithm with those from the Bretagnon \& Francou (1988) algorithm and also with those from a third independent program kindly provided by F. Chollet (private communication). In all cases the velocity difference was below $1 \mathrm{~ms}^{-1}$.

Two standard templates or masks are available. They have been built following the Baranne et al. (1979) technique, using Bell \& Gustafsson synthetic spectra of an F0V and K0III star.

Many Monte-Carlo simulations with ELODIE spectra were carried out to investigate the error in the parameters of the CCF fitted by Gaussians due to photon noise and CCD read-out noise. With the help of the Eq. (10) of Queloz (1995b) a similar expression has been computed and calibrated specifically for ELODIE. The uncertainty in the radial velocity and the width of the $\mathrm{CCF}$ due to the photon noise is:

$$
\begin{gathered}
\varepsilon_{\mathrm{p}}\left(v_{\mathrm{r}}\right)=\frac{C\left(T_{\mathrm{eff}}\right)}{D S / N} \cdot \frac{(1+0.2 w)}{3}\left(\mathrm{~km} \mathrm{~s}^{-1}\right), \\
\varepsilon_{\mathrm{p}}(\mathrm{FWHM})=2.355 \cdot \varepsilon_{\mathrm{p}}\left(v_{\mathrm{r}}\right)\left(\mathrm{km} \mathrm{s}^{-1}\right),
\end{gathered}
$$

where $C\left(T_{\text {eff }}\right)$ is a constant which depends on the spectral type of the star and on the mask used, $w$ the (noiseless) FWHM (in $\mathrm{km} \mathrm{s}^{-1}$ ) of the CCF, $D$ its (noiseless) relative depth and $S / N$ the mean signal-to-noise ratio. The $C\left(T_{\text {eff }}\right)$ parameter has been computed from MonteCarlo simulations for both standard masks using ELODIE spectra of $\mathrm{G}, \mathrm{K}$ and $\mathrm{M}$ dwarfs (Table 2). The parameter $C\left(T_{\text {eff }}\right) / D$ is inversely proportional to the relative efficiency of each mask. It is clear from Table 2 that the best efficiency is reached when the spectrum is matched by the template ( $\mathrm{F}$ mask for a $\mathrm{F}$ star, etc.).

In the case of a "star fibre and sky fibre" exposure, the CCF of the star is automatically corrected for the sky $\mathrm{CCF}$ (due to the moon light). The sky CCF is computed using the same wavelength limits and the same template spectrum as for the star CCF. If a Gaussian can be reasonably fitted to the sky CCF, the fit is subtracted from the CCF of the star (see Fig. 15); otherwise, only the mean level of the sky-CCF is used. 
Table 2. Value of the $C\left(T_{\text {eff }}\right)$ constant of Eq. (9). The $C\left(T_{\text {eff }}\right) / D$ parameter is a inverse measurement of the mask efficiency

\begin{tabular}{lllll}
\hline star $T_{\text {eff }}$ & \multicolumn{2}{c}{$C\left(T_{\text {eff }}\right)$} & \multicolumn{2}{c}{$C\left(T_{\text {eff }}\right) / D$} \\
& F mask & K mask & F mask & K mask \\
\hline 4000 & 0.09 & 0.050 & 0.45 & 0.29 \\
5000 & 0.08 & 0.040 & 0.30 & 0.27 \\
6000 & 0.07 & 0.045 & 0.29 & 0.37 \\
\hline
\end{tabular}

Fig. 15. a) CCF of a faint star contaminated by the CCF of the solar spectrum (indicated by an arrow). b) Same CCF after the subtraction of the CCF of the sky. The line is the Gaussian fit

\section{Performances}

We measure, with the thorium emission lines, a sampling per resolution element (FWHM) of $2.25 \pm 0.2$ (pixels) in the blue and $2.1 \pm 0.2$ (pixels) in the red. After wavelength calibration, the width of the lines (FWHM) is $7.4 \mathrm{~km} \mathrm{~s}^{-1}$ in the blue and $7.0 \mathrm{~km} \mathrm{~s}^{-1}$ in the red corresponding to an average resolution power of 42000 . Figure 16 displays a part of the ELODIE spectrum of the G0V star HD109358.

During good quality nights, in one hour exposure time, a $S / N$ of 100 per pixel at $\lambda=5600 \AA$ can be achieved for a star of $m_{V}=9.75$. This corresponds to a global transmission of $1.3 \%$. Taking into account $60 \%$ transmission for the atmosphere and the telescope, $65 \%$ for the CCD and $1.5^{\prime \prime}$ seeing, this corresponds to $4.2 \%$ efficiency for ELODIE alone.
Fig. 16. Part of the spectrum of the G0V star HD109358 (centre of the 117th order)

The relative sensitivity of ELODIE displayed in Fig. 17 indicates a severe loss of efficiency in the blue part of the spectrum. This effect is partly due to the CCD response but is, in our case, strengthened by the blaze response of the grism, which has its maximum efficiency at $5500 \AA$ and therefore has a poor performance in the blue domain.

Fig. 17. Relative efficiency of ELODIE to $5600 \AA$, over the whole wavelength range, computed from the observation of the spectro-photometric OV star HD93521. The squares represents the relative quantum efficiency of the CCD

The evaluation of the spectroscopic quality of ELODIE spectra was made by comparison of the observed equivalent widths in the solar spectrum with the values of the Moore (1966) atlas. Using a spectrum of a minor planet (Vesta) and a set of 221 isolated lines, the following regression was measured: $W_{\lambda}($ Moore $)=0.975 \cdot W_{\lambda}($ ELODIE $)+$ 
0.6 (mA) (see Fig. 18). The small value of the zero point $(0.6 \mathrm{~m} \AA)$ indicates that only a little background contamination remains in this spectrum. The slope is not equal to unity, but a similar result is obtained for the MUSICOS echelle spectrograph (Baudrand \& Böme 1992) in spite of the use of a more sophisticated background modelling.

Fig. 18. Comparison between the ELODIE equivalent widths and the Moore (1966) results for the solar spectrum. The line represents $W_{\lambda}$ (Moore) $=W_{\lambda}($ ELODIE $)$

The stability of the spectrograph in wavelength during the night depends on many parameters. The strongest variability $\left(\Delta V_{\mathrm{r}} \sim 300 \mathrm{~m} \mathrm{~s}^{-1}\right)$ occurs during the filling of the dewar but, after 45 minutes, the effects of this strong thermal shock vanish. During the night, ELODIE is little dependent on the temperature because its thermal inertia is high enough and also because the spectrograph room is thermostated. The main remaining influence is the atmospheric pressure that changes the air refractive index and shifts the zero of the calibration $\left(100 \mathrm{~m} \mathrm{~s}^{-1} \mathrm{mmHg}^{-1}\right)$. There is also a small flexure effect due to the dewar unloading which moves the orders in the direction perpendicular to the main dispersion. The orders are vertically shifted by about 0.1 pixels during the night and the effect seen on the wavelength calibration is about $50 \mathrm{~m} \mathrm{~s}^{-1}$.

\subsection{Towards higher accuracy in radial velocity}

A monitoring of the wavelength variation is compulsory to achieve radial velocity measurements with an accuracy better than $50 \mathrm{~m} \mathrm{~s}^{-1}$. Such measurements are done using the sky-hole illuminated by the thorium lamp during stellar exposures. The wavelength solutions of each optical path (star and sky) are independent, but series of tests have shown that their variations are strongly correlated. Therefore, the sky-way can be used to monitor the wavelength calibration shift of the star-way.
Fig. 19. a) histogram of the velocity shifts between the two way (sky fibre - star fibre). The line is a Gaussian with $\sigma=2.3 \mathrm{~ms}^{-1}$. Note that a correction is done to suppress a slight mismatch between the two optical paths: $\Delta V_{\mathrm{r}}(\mathrm{sky})=\Delta V_{\mathrm{r}}(\mathrm{star})-.04 \Delta V_{\mathrm{r}}(\mathrm{star})+0.6\left(\Delta V_{\mathrm{r}}\right.$ is the velocity variation of the spectrograph in units of $\left.\mathrm{m} \mathrm{s}^{-1}\right)$. b) histogram of the variation of the spectrograph monitor by the star-way during the measurements

During 2 nights, 40 thorium exposures were carried out. In order to simulate the worst experimental situation, we also made a serie of thermal shocks in the spectrograph room ( $\Delta T \sim 1$ degree). Under such conditions, the velocity variations due to the calibration shift between 2 successive exposures sometimes reached $150 \mathrm{~m} \mathrm{~s}^{-1}$. However, despite the strong variability of the spectrograph during this experiment, we observe that the velocity variations between both optical paths (star and sky) are very small. Using the first exposure of the series as a wavelength calibration for each way, the velocity differences between the ways, for each successive exposure, exhibit only a $2.5 \mathrm{~m} \mathrm{~s}^{-1}$ standard deviation (see Fig. 19). Note that a small velocity correction is taken into account to correct a slight mismatch between the two optical paths. Finally, by adding the uncertainty in the wavelength calibration of the first exposure, we conclude that ELODIE is able to provide an accuracy of about $4 \mathrm{~m} \mathrm{~s}^{-1}$ with this technique.

The investigation of the short-term radial velocity measurement accuracy has been carried out with the bright star HD109358 (G0V). Successively, during 3 hours, 13 stellar exposures (simultaneous thorium type), of 7 minutes each, were obtained. The individual velocities show a standard deviation of $6 \pm 1 \mathrm{~m} \mathrm{~s}^{-1}$. As the signal-tonoise ratio of each spectrum is high enough $(S / N>150)$ to neglect the photon-noise errors, this corresponds to the short-term radial velocity instrumental uncertainty of ELODIE. However, to search for planets or brown dwarfs by Doppler shift, the individual radial velocity accuracy 
Table 3. Summary of the ELODIE instrumental characteristics

\begin{tabular}{|c|c|}
\hline Instrumental parameter & measured value \\
\hline Typical resolution power & $42^{\prime} 000$ \\
\hline Pixel sampling per FWHM & $1.9-2.45$ \\
\hline Usable wavelength domain (blaze response $>50 \%$ ) & $3906-6811 \AA$ \\
\hline Number of orders & 67 \\
\hline Typical vertical width of the orders ( $90 \%$ of the energy) & 3 pixels \\
\hline CCD read-out noise & $8.5 \mathrm{e}^{-}$ \\
\hline Overall transmission at $5600 \AA$ (maximum of the blaze) & $1.3 \%$ \\
\hline Internal precision of the wavelength calibration & $3 \mathrm{~m} \mathrm{~s}^{-1}\left(20 \mathrm{~m} \mathrm{~s}^{-1}\right.$ per order $)$ \\
\hline Observed instrumental velocity error & $13 \mathrm{~ms}^{-1}$ (long term) \\
\hline
\end{tabular}

over the long term is a more crucial parameter. Ideally, the long term accuracy should be close to the short term accuracy, but this is usually not the case because of long term instabilities of the spectrograph and of the shift of the lines of the star over the detector during the year.

To measure accurately and with objectivity the value of the long term radial velocity accuracy of ELODIE, we have used all the radial velocities measured since April 1994 as a part of the sub-stellar companion search program (Mayor et al. 1996). For a set of stars with constant velocities, we expect the $P\left(\chi^{2}\right)$ distribution to be flat. Thus, the value of the instrumental error can be tuned up to meet this statistical property (Duquennoy et al. 1991b). Using 106 stars of the sample, measured at least twice, we find, using this technique, an instrumental uncertainty of $13 \mathrm{~m} \mathrm{~s}^{-1}$. This result is excellent by comparison with the performances achieved by other groups (see Kürster et al. 1994).

The instrumental long term radial velocity uncertainty is significatively larger than the short term accuracy. However, note that for ELODIE a velocity shift of $12 \mathrm{~m} \mathrm{~s}^{-1}$, at CCD scale, corresponds to $1 / 300$ of the pixel size. Jorden et al. (1994) show that at this scale the CCD is strongly non-uniform and each pixel has an intrinsic uncertainty on its true centre of gravity. Considering 4000 lines spread over 5 pixels and an uncertainty of $1 / 4$ pixel in the location of each pixel, with simple statistics, we find an error in the average location of $0.25 / \sqrt{4000 \cdot 5}=1 / 600$ pixel corresponding to $6 \mathrm{~m} \mathrm{~s}^{-1}$. The addition of this uncertainty to the short term accuracy computed above shows that part of the long term accuracy is certainly due to this effect. The use of a CCD with smaller size pixels could be an easy way to improve the long term accuracy.

\section{Summary}

We have described ELODIE, the new spectrograph of the Haute-Provence Observatory, a fibre-fed echelle spectrograph with a fixed optical system, designed to provide accurate radial velocity measurements. The main disper- sion is done by a $\tan \theta=4$ echelle-grating and the crossdispersion by the combination of a prism and a grism. This special optical mounting allows us to record in one single exposure a wavelength domain ranging from 3906 $\AA$ to $6811 \AA$ with a resolution of 42000 on a relatively small CCD $(1024 \times 1024)$. An automatic data reduction program, integrated into the spectrograph data flow, reduces all images directly after the exposure. The software also includes the computation of cross-correlation functions. This very original ability proves to be very useful to interact rapidly with the observations (in particularly for the search for small velocity variations). It also saves the observer the labour of reducing echelle spectra. A summary of the instrumental characteristics of ELODIE is presented in Table 3.

The efficiency of ELODIE allows us to observe stars of 9th-magnitude class for spectroscopic works. During good nights, observations of 16th-magnitude stars are also possible to measure for example the radial velocity at $1 \mathrm{~km} \mathrm{~s}^{-1}$ precision by cross-correlation. We clearly see that the potential research field of this instrument is very wide, e.g.: stellar atmosphere physics (pulsating stars, abundances studies), Galactic studies (velocity, metallicity, $\left.T_{\text {eff }}\right)$, study of clusters, pre-MS stars (membership, binarity, rotation) and binary star studies.

The long term radial velocity instrumental uncertainty of ELODIE is $13 \mathrm{~m} \mathrm{~s}^{-1}$. This indicates that our technique of simultaneously monitoring the spectrograph by using a second fibre, illuminated by a thorium-argon lamp, is able to provide similar performances to that of absorption cell instruments. With its wide wavelength range and its relatively good transmission, ELODIE can measure the velocity of a solar type star of 9 th magnitude, with $5 \mathrm{~m} \mathrm{~s}^{-1}$ photon error, in less than 30 minutes. It is then possible to monitor a large number of stars, using a reasonable amount of telescope time. ELODIE is a suitable instrument for hunting Jupiter size planet around nearby stars $^{3}$

\footnotetext{
${ }^{3}$ After submission of this paper, the first jovian planet orbiting a solar-type star has been discovered with the spectrograph ELODIE (Mayor \& Queloz 1995).
} 
A copy of this spectrograph called CORALIE will be installed at La Silla (ESO, Chili) on the new 1.20-meter telescope of the Geneva Observatory. CORALIE is almost identical to ELODIE. However, the smaller focal length of this telescope, compared to the $193 \mathrm{~cm}$ of the OHP, will increase the resolution up to 50000 . The detector is also different. It has smaller pixels and will provide a better sampling (3 instead of 2 pixels per resolution element). We expect that all these modifications will improve the accuracy of the radial velocities.

Acknowledgements. We are extremely grateful to the region "Provence Alpes Côtes d'Azur" (PACA) for its continuous support of this project. In particular, the design of the instrument was only made possible thanks to grants offered by this region. The two spectrographs of this project have been financed by the PACA, the INSU (France), the University of Geneva and by the FNRS (Switzerland). We are thankful to these organizations for their confidence and generous support. We have benefited from the invaluable help of many colleagues. We present our particular acknowledgements to Catherine Grezes-Besset of the Ecole de Physique de Marseille, for having developed the filter located in front of the CCD. We also thank Luc Weber of the Geneva Observatory for providing us with important items of software and for his continuous and enthusiastic support. We are also grateful to Raphael Cautain for his contribution to the data reduction software, to Denis Gillet and F. Chollet for their contribution to tests and improvement of the algorithm of the Earth velocity correction, to Frédéric Pont for his careful reading of the manuscript and to John Hearnshaw, our referee, for his comments and suggestions. We finally thank Philippe Véron, former director of the OHP, for his strong support along all the critical phases of this project.

\section{References}

Avila G., 1988, ASP Conf. Ser. 13, 63

Babel J., North P., Queloz D., 1995, A\&A 300, 15

Baranne A., Mayor M., Poncet J.L., 1977, C.R. Acad. Sc. Paris 260, 3283

Baranne A., Mayor M., Poncet J.L., 1979, Vistas astron. 23, 279

Baudrand J., Böme T., 1992, A\&A 259, 711

Benz W., Mayor M., 1981, A\&A 93, 235

Benz W., Mayor M., 1984, A\&A 138, 183

Bretagnon P., Francou G., 1998, A\&A 202, 309

Campbell B., Walker G.A.H., 1979, PASP 91, 540

Cochran W.D., 1988, ApJ 334, 349

Cochran W.D., Hatzes A.P., 1990, in "Optical Spectroscopic Instrumentation and Techniques for the 1990's". In:
McNamara B.J. and Lerner J.M. (eds.), Proc. SPIE 1318, 148

Connes P., 1985, Ap\&SS 110, 211

Dubath P., Meylan G., Mayor M., Magain P., 1990, A\&A 239, 142

Duquennoy A., Mayor M., 1991a, A\&A 248, 485

Duquennoy A., Mayor M., Halbwachs J.-L., 1991b, A\&AS 88, 281

Griffin R., 1967, ApJ 148, 465

Griffin R., Griffin R., 1973, MNRAS 162, 243

Hall J.C., Fulton E.E., Huenemoerder D.P, Welty A.D., Neff J.E., 1994, PASP 106, 315

Heacox W., 1986, AJ 92, 219

Horn K., 1986, PASP 98, 609

Jorden P.R., Delton J.-M., Oates A.P., 1994, "Instrumentation in Astronomy VIII", 2198, 57

Kürster M, Hatzes A.P., Cochran W.D., et al., 1994, The Messenger 76, 51

Marcy G.W., Williams E., Mao L., 1993, in "Remote Sensing Reviews", Vol. 8, 202

Marcy G.W., Butler R.P., 1994, in ESO workshop "The bottom of the main sequence and beyond". In: Tinney G. (ed.). Springer Verlag, Heidelberg, p. 98

Mayor M., 1980, A\&A 87, L1

Mayor M., Queloz D., 1995, Nat 378, 355

Mayor M., Queloz D., 1996, in $9^{\text {th }}$ Cambridge Workshop: "Cool Stars, Stellar Systems, and the Sun" (in press)

McMillan R.S., Smith P.H., Perry M.S., Moore T.L., Merline W.J., 1990, in "Instrumentation in Astronomy VII". In: Crawford (D.L.), Proc. SPIE 1235, 601

Moore C.E, Minnaert M.G.I., Houtgast J., 1966, NBS, Monograph 61

Murdoch K.A., Hearnshow J.B., Clark M.A., 1993, ApJ 413, 349

Palmer B.A., Engleman R.Jr., 1983, Atlas of the Thorium Spectrum, Los Alamos National Laboratory. In: Sinoradzki H. (ed.)

Pont F., Queloz D., Mayor M., Burki G., 1995a, in IAU Symposium 169 "Unsolved Problems of the Milky Way". In: Blitz L. (ed.) (in press)

Pont F., Queloz D., Mayor M., Bratschi P., 1995b, in IAU Coll. 155, "Astrophysical Applications of Stellar Pulsation", ASP Conf. Ser. 83. In: Stobie R.S., Whitelock P.A. (eds.), p. 194

Queloz D., Dubath P., Pasquini L., 1995a, A\&A 300, 31

Queloz D., 1995b, in IAU Symposium 167, "New Developments in Array Technology and Applications". In: Davis Philip A.G. (ed.). Dordrecht: Kluwer, p. 221

Queloz D., Babel J., Mayor M., Bouvier J., 1996, in $9^{\text {th }}$ Cambridge Workshop: "Cool Stars, Stellar Systems, and the Sun" (in press)

Stumpff P., 1980, A\&AS 41, 1

Walker G.A.H., Walker A.R., Irwin A.W., et al., 1995, Icarus 116,359 